TAIWANESE JOURNAL OF MATHEMATICS

Vol. 3, No. 1, pp. 115-122, March 1999

\title{
ON THE GLIDING HUMPS PROPERTY
}

\author{
Li Ronglu and Min-Hyung Cho
}

\begin{abstract}
We establish two uniform convergence results for duality pairs consisting of vector-sequence spaces with some gliding humps property and the corresponding function-sequence spaces. These uniform convergence results imply some important facts.
\end{abstract}

Let $\mathrm{X}, \mathrm{Y}$ be topological vector spaces and $E(X)$ a vector space of $\mathrm{X}$-valued sequences. For $x \in E(X)$, let $x_{k}$ denote the $k^{\text {th }}$ coordinate of $x$ and, hence, if $\left\{x^{n}\right\}$ is a sequence in $E(X)$, then $x_{k}^{n}$ is just the $k^{\text {th }}$ coordinate of the $n^{\text {th }}$ vector-sequence $x^{n} \in E(X)$.

Let $\left[Y^{E(X)}\right]^{\beta}$ be the family of function-sequences $\left\{f_{k}\right\} \subseteq Y^{X}$ for which $f_{k}(0)=0$ for all $k$ and the series $\sum_{k=1}^{\infty} f_{k}\left(x_{k}\right)$ converges in $Y$ for each $x=\left(x_{k}\right) \in$ $E(X)$. Especially, let $E(X)^{\beta Y}=\left\{T=\left(T_{k}\right) \in\left[Y^{E(X)}\right]^{\beta}\right.$ : each $T_{k}$ is linear and continuous\} (see $[10,11,13,14,15,17])$. As usual, for $f=\left(f_{k}\right) \in\left[Y^{E(X)}\right]^{\beta}$ and $x=\left(x_{k}\right) \in E(X)$, we write $f \cdot x=\sum_{k=1}^{\infty} f_{k}\left(x_{k}\right)=\sum_{k=1}^{\infty} f_{k} x_{k}$.

Throughout this paper we assume that $E(X) \supseteq c_{00}(X)=\left\{x=\left(x_{k}\right) \in\right.$ $X^{\mathbb{N}}: x_{k}=0$ eventually $\}$ and $E(X)$ is equipped by some Hausdorff vector topology which is stronger than the topology of coordinatewise convergence and, hence, $E(X)$ is a $K(X)$ space [3]. Let $P_{n}: E(X) \rightarrow E(X)$ be the section map which sends $\left(x_{1}, x_{2}, \ldots\right)$ to $\left(x_{1}, \ldots, x_{n}, 0,0, \ldots\right)$. We say that $E(X)$ has the property SUB if $\left\{P_{n}\right\}_{n=1}^{\infty}$ is uniformly bounded on bounded subsets of the domain space $E(X)[13]$.

Following D. Noll [10], we say that a sequence $\left\{z^{n}\right\}$ of nonzero vectors in $E(X)$ is a block sequence if there is a strictly increasing $\left\{k_{n}\right\} \subseteq \mathbb{N}$ such that $z^{n}=\left(0, \ldots, 0, z_{k_{n}+1}^{n}, z_{k_{n}+2}^{n}, \ldots, z_{k_{n+1}}^{n}, 0,0, \ldots\right)$. We say that $E(X)$ has

Received September 3, 1997; revised November 13, 1997.

Communicated by P. Y. Wu.

1991 Mathematics Subject Classification: 46E40.

Key words and phrases: The gliding humps property, boundedly continuous function, uniform convergence. 
the strong gliding humps property (SGHP) if every bounded block sequence $\left\{z^{n}\right\}$ in $E(X)$ has a subsequence $\left\{z^{n_{i}}\right\}$ such that the coordinatewise sum $\sum_{i=1}^{\infty} z^{n_{i}}=\left(0, \ldots, 0, z_{k_{n_{1}}+1}^{n_{1}}, \ldots, z_{k_{n_{1}+1}}^{n_{1}}, 0, \ldots, 0, z_{k_{n_{2}}+1}^{n_{2}}, \ldots, z_{k_{n_{2}+1}}^{n_{2}}, 0, \ldots\right)$ belongs to $E(X)[13]$. We say that $E(X)$ has the zero gliding humps property (0-GHP) if for $x^{n} \rightarrow 0$ in $E(X)$ and $i_{1} \leq j_{1}<i_{2} \leq j_{2}<\cdots$ in $\mathbb{N}$, there is an increasing $\left\{n_{p}\right\} \subseteq \mathbb{N}$ such that $\left(0, \ldots, 0, x_{i_{n_{1}}}^{n_{1}}, \ldots, x_{j_{n_{1}}}^{n_{1}}, 0, \ldots, 0, x_{i_{n_{2}}}^{n_{2}}, \ldots, x_{j_{n_{2}}}^{n_{2}}, 0, \ldots\right)$ belongs to $E(X)$. This form of the gliding humps property was introduced in Lee Peng Yee [5]; see also [6], [14]. For a discussion of various forms of gliding humps properties and their applications, see [2].

$F \subseteq\left[Y^{E(X)}\right]^{\beta}$ is said to be conditionally $w E(X)$-sequentially compact if every sequence $\left\{f^{n}\right\} \subseteq F$ has a subsequence $\left\{f^{n_{i}}\right\}$ such that $\lim f^{n_{i}} \cdot x$ exists at each $x \in E(X)$. Finite subsets of $\left[Y^{E(X)}\right]^{\beta}$ are conditionally $w E(X)$ sequentially compact, and if $F$ is sequentially compact under the topology $w E(X)$ of pointwise convergence on $E(X)$, then $F$ is conditionally $w E(X)$ sequentiaiiy compact. In this paper we would like to establish two uniforn convergence results for conditionally $w E(X)$-sequentially compact subfamilies of $\left[Y^{E(X)}\right]^{\beta}$ whenever $E(X)$ has some properties such as SUB, SGHP and 0GHP, etc. Using these uniform convergence theorems we shall consummate and improve some recent results in [13], [14], [17].

Theorem 1. Let $E(X)$ be a vector-sequence space having $S U B$ and $S G H P$. If a subfamily $F$ of $\left[Y^{E(X)}\right]^{\beta}$ is conditionally $w E(X)$-sequentially compact, then for every bounded $B \subseteq E(X)$, the series $\sum_{k=1}^{\infty} f_{k} x_{k}$ converges uniformly for $f \in F$ and $x=\left(x_{k}\right) \in B$.

Proof. If not, there exists a neighborhood $U$ of $0 \in Y$ such that if $n_{0} \in \mathbb{N}$ then $\sum_{k=n}^{\infty} f_{k} x_{k} \notin U$ for some $n>n_{0}, f \in F$ and $x \in B$. Thus, there exist $n_{1}>1, f^{1} \in F$ and $x^{1} \in B$ such that $\sum_{k=n_{1}}^{\infty} f_{k}^{1} x_{k}^{1} \notin U$. Pick a neighborhood $V$ of $0 \in Y$ for which $V+V \subseteq U$. Since the series $\sum_{k=1}^{\infty} f_{k}^{1} x_{k}^{1}$ converges, there is an $m_{1}>n_{1}$ for which $\sum_{k=n_{1}}^{m_{1}} f_{k}^{1} x_{k}^{1} \notin V$. Similarly, there exist integers $m_{2}>n_{2}>m_{1}, f^{2} \in F$ and $x^{2} \in B$ such that $\sum_{k=n_{2}}^{m_{2}} f_{k}^{2} x_{k}^{2} \notin V$. Continuing this construction, we have an integer sequence $n_{1}<m_{1}<n_{2}<m_{2}<\cdots,\left\{f^{i}\right\} \subseteq F$ and $\left\{x^{i}\right\} \subseteq B$ such that

$$
\sum_{k=n_{i}}^{m_{i}} f_{k}^{i} x_{k}^{i} \notin V, i=1,2,3, \ldots
$$


Let $z^{j}=\left(0, \ldots, x_{n_{j}}^{j}, \ldots, x_{m_{j}}^{j}, 0,0, \ldots\right)$. Since $E(X)$ has SUB and $B$ is a bounded subset of $E(X)$, the set $\left\{P_{n} x-P_{m} z: n, m \in \mathbb{N}, x, z \in B\right\}$ is bounded and, hence, $\left\{z^{j}\right\}$ is a bounded block sequence in $E(X)$.

Now consider the matrix $\left(f^{i} \cdot z^{j}\right)_{i, j}$. Since $F$ is conditionally $w E(X)$ sequentially compact, by passing to a subsequence if necessary, we may assume that $\lim _{i} f^{i} \cdot x$ exists at each $x \in E(X)$ and, hence, $\lim _{i} f^{i} \cdot z^{j}$ exists for all $j$. Let $j_{1}<j_{2}<\ldots$ in $\mathbb{N}$. Then there is a subsequence $\left\{j_{p_{r}}{ }^{i}\right\} \subseteq\left\{j_{p}\right\}$ such that the sequence $\left(0, \ldots, 0, x_{n_{p_{1}}}^{j_{p_{1}}}, \ldots, x_{m_{j_{1}}}^{j_{p_{1}}}, 0, \ldots, 0, x_{n_{j_{p_{2}}}}^{j_{p_{2}}}, \ldots, x_{m_{j_{p_{2}}}}^{j_{p_{2}}}, 0, \ldots, 0, \ldots\right)$ belongs to $E(X)$ because $E(X)$ has SGHP, i.e., the coordinatewise sum $z=\sum_{r=1}^{\infty} z^{j_{p_{r}}}$ belongs to $E(X)$. Hence, for each $i$ we have $f^{i} \cdot z=\sum_{r=1}^{\infty} \sum_{k=n_{j_{p_{r}}}}^{m_{j_{p_{r}}}} f_{k}^{i} x_{k}^{j_{p_{r}}}=\sum_{r=1}^{\infty} f^{i} . z^{j_{p_{r}}}$ and $\lim _{i} \sum_{r=1}^{\infty} f^{i} \cdot z^{j_{p_{r}}}=\lim _{i} f^{i} \cdot z$ exists. Thus, by the Antosik-Mikusinski matrix theorem [1], [8], $\lim _{i} \sum_{k=n_{i}}^{m_{i}} f_{k}^{i} x_{k}^{i}=\lim _{i} f^{i} \cdot z^{i}=0$. This contradicts $(*)$.

Note that, in Theorem 1, each mapping $f=\left(f_{k}\right) \in F$ need not be linear and each coordinate function $f_{k}$ need not be continuous. In fact, we only require that $f \cdot 0=0$, i.e., $f_{k}(0)=0$ for all $k$, while [13], [14], [17] gave results only for continuous linear $f_{k}: X \rightarrow Y$ and $f=\left(f_{k}\right) \in E(X)^{\beta Y}$. A similar improvement to Schur's theorem was given in [9] for abstract function matrices.

Corollary 2. ([13], Th. 4). Assume that $E(X)$ has properties $S U B$ and $S G H P$ and that $X$ is an $\mathcal{A}$-space. If $F \subseteq E(X)^{\beta Y}$ is pointwise bounded on $E(X)$, then $F$ is uniformly bounded on bounded subsets of $E(X)$.

Proof. Let $\left\{x^{n}\right\}$ be a bounded sequence in $E(X)$ and $\left\{f^{n}\right\} \subseteq F$. Since $E(X)$ has SUB, for each $k,\left\{\left(0, \ldots, 0, x_{k}^{n}, 0,0, \ldots\right): n \in \mathbb{N}\right\}$ is bounded in $E(X)$ and, since the topology on $E(X)$ is stronger than the topology of coordinatewise convergence, $\left\{x_{k}^{n}\right\}_{n=1}^{\infty}$ is bounded in $X$ and $\lim _{n} \frac{1}{n} f_{k}^{n} x_{k}^{n}=0$ because $X$ is an $\mathcal{A}$-space ([8], Cor. 4). On the other hand, $\left\{f^{n}\right\}$ is pointwise bounded on $E(X)$ and, hence, $\frac{1}{n} f^{n} \cdot x \rightarrow 0$ at each $x \in E(X)$, i.e., the set $\left\{\frac{1}{n} f^{n}: n \in \mathbb{N}\right\}$ is conditionally $w E(X)$-sequentially compact. Now by Theorem 1 the series $\sum_{k=1}^{\infty} \frac{1}{n} f_{k}^{n} x_{k}^{m}$ converges uniformly for $n, m \in \mathbb{N}$. Thus,

$$
\lim _{n} \frac{1}{n} f^{n} \cdot x^{n}=\lim _{n} \sum_{k=1}^{\infty} \frac{1}{n} f_{k}^{n} x_{k}^{n}=\sum_{k=1}^{\infty} \lim _{n} \frac{1}{n} f_{k}^{n} x_{k}^{n}=0 .
$$

Using 0-GHP instead of SGHP, C. Swartz gave a similar but more clear-cut uniform boundedness result; see [15], Th. 12.5.7. 
We say that a map $u: X \rightarrow Y$ is boundedly continuous if $\left(x_{a}\right)$ is a bounded net in $X$ such that $x_{a} \rightarrow x_{0}$ in $X$, then $u\left(x_{a}\right) \rightarrow u\left(x_{0}\right)$. Clearly, a continuous map is boundedly continuous and a boundedly continuous map must be sequentially continuous. Theorem 1 of [13] asserts that if $E(X)$ has some suitable properties, then each $f=\left(f_{k}\right) \in E(X)^{\beta Y}$ is sequentially continuous. Note that if $f=\left(f_{k}\right) \in E(X)^{\beta Y}$, then each coordinate function $f_{k}$ is continuous and linear. In contrast to Theorem 1 of [13], the following result only requires that coordinate functions be boundedly continuous.

Corollary 3. Assume that $E(X)$ has $S U B$ and $S G H P$. If $f=\left(f_{k}\right) \in$ $\left[Y^{E(X)}\right]^{\beta}$ is such that each coordinate function $f_{k}: X \rightarrow Y$ is boundedly continuous, then $f$ is boundedly continuous on $E(X)$ and, hence, $f$ is sequentially continuous. In particular, each $T=\left(T_{k}\right) \in E(X)^{\beta Y}$ is boundedly continuous and, hence, $T$ is sequentially continuous.

Proof. Let $\left(x^{a}\right)_{a \in I}$ be a bounded net such that $x^{a} \rightarrow x$ in $E(X)$. Then for each $k$ we have $x_{k}^{a} \rightarrow x_{k}$ because the topology on $E(X)$ is stronger than the topology of coordinatewise convergence. Since $E(X)$ has SUB, for each $k$ the net $\left(x_{k}^{a}\right)_{a \in I}$ is bounded in $X$. Thus, for each $k, \lim f_{k} x_{k}^{a}=f_{k} x_{k}$ by hypothesis. Since the singleton $\{f\}$ is conditionally $w E(X)$-sequentially compact and $\left(x^{a}\right)_{a \in I}$ is bounded, by Theorem 1 the series $\sum_{k=1}^{\infty} f_{k} x_{k}^{a}$ converges uniformly for $a \in I$. Therefore,

$$
\lim _{a} f \cdot x^{a}=\lim _{a} \sum_{k=1}^{\infty} f_{k} x_{k}^{a}=\sum_{k=1}^{\infty} \lim _{a} f_{k} x_{k}^{a}=\sum_{k=1}^{\infty} f_{k} x_{k}=f \cdot x .
$$

If $E(X)$ has 0 -GHP, then for every $T=\left(T_{k}\right) \in E(X)^{\beta Y}$ and $x^{n} \rightarrow 0$ in $E(X)$, the series $\sum_{k=1}^{\infty} T_{k} x_{k}^{n}$ converges uniformly for $n \in \mathbb{N}$ ([17], Th. 2). We would like to establish a similar result for $F \subseteq\left[Y^{E(X)}\right]^{\beta}$. Recall that $E(X)$ is an AK-space if $\left(0, \ldots, 0, x_{n}, x_{n+1}, \ldots\right) \rightarrow 0$ for each $\left(x_{k}\right) \in E(X)([15]$, p. 128). For example, the sequence spaces $\left(c_{0},\|\cdot\|_{\infty}\right)$ and $\left(1^{p},\|\cdot\|_{p}\right)$ are AK-spaces having $0-$ GHP.

Theorem 4. Let $E(X)$ be an AK-space with 0-GHP. If $F$ is a conditionally $w E(X)$-sequentially compact subfamily of $\left[Y^{E(X)}\right]^{\beta}$, then for every $x^{n} \rightarrow 0$ in $E(X)$ the series $\sum_{k=1}^{\infty} f_{k} x_{k}^{n}$ converges uniformly with respect to $f=\left(f_{k}\right) \in F$ and $n \in \mathbb{N}$.

Proof. Firat we claim that for each $x=\left(x_{k}\right) \in E(X)$ the series $\sum_{k=1}^{\infty} f_{k} x_{k}$ converges uniformly with respect to $f=\left(f_{k}\right) \in F$. If not, there exist a neighborhood $V$ of $0 \in Y,\left\{f^{i}\right\} \subseteq F$ and an integer sequence $n_{1}<m_{1}<n_{2}<m_{2}<$ 
$\ldots$ such that $\sum_{k=n_{i}}^{m_{i}} f_{k}^{i} x_{k} \notin V$ for all i. If $z^{j}=\left(0, \ldots, 0, x_{n_{j}}, \ldots, x_{m_{j}}, 0,0, \ldots\right)$, then $z^{j} \rightarrow 0$ because $E(X)$ is an AK-space. Since $F$ is conditionally $w E(X)$ sequentially compact, by passing to a subsequence if necessary, we may assume that $\lim _{i} f^{i} \cdot x$ exists at each $x \in E(X)$. As in the proof of Theorem 1 , by 0 GHP, the Antosik-Mikusinski theorem shows that $\sum_{k=n_{i}}^{m_{i}} f_{k}^{i} x_{k} \rightarrow 0$, but this is a contradiction.

Now suppose the conclusion of Theorem 4 fails. Then there exists a neighborhood $V$ of $0 \in Y$ satisfying

(*) $\forall n_{0} \in \mathbb{N} \exists m>n>n_{0}, f \in F$ and $i \in \mathbb{N}$ such that $\sum_{k=n}^{m} f_{k} x_{k}^{i} \notin V$.

Thus, there exist $m_{1}>n_{1}>1, f^{1} \in F$ and $i_{1} \in \mathbb{N}$ such that $\sum_{k=n_{1}}^{m_{1}} f_{k}^{1} x_{k}^{i_{1}} \notin V$. By the first part of this proof, there is an $m_{0} \in \mathbb{N}$ such that $\sum_{k=n}^{m} f_{k} x_{k}^{i} \in V$ for all $m \geq n>m_{0}, f=\left(f_{k}\right) \in F$ and $1 \leq i \leq i_{1}$. Therefore, by $(*)$ again, there exist $m_{2}>n_{2}>\max \left(m_{0}, m_{1}\right), f^{2} \in F$ and $i_{2}>i_{1}$ such that $\sum_{k=n_{2}}^{m_{2}} f_{k}^{2} x_{k}^{i_{2}} \notin V$. Continuing this construction we have integer sequences $n_{1}<m_{1}<n_{2}<$ $m_{2}<\ldots, i_{1}<i_{2}<\cdots$ and $\left\{f^{p}\right\} \subseteq F$ such that $\sum_{k=n_{p}}^{m_{p}} f_{k}^{p} x_{k}^{i_{p}} \notin V$ for all $p \in \mathbb{N}$. However, as before the Antosik-Mikusinski theorem, the condition 0-GHP, and $x^{i_{p}} \rightarrow 0$ imply a contradictory fact.

Now we would like to show some applications of Theorems 1 and 4 . lt is easy to see that with the norm $\left\|\left(t_{k}\right)\right\|_{\infty}=\sup _{k}\left|t_{k}\right|$, the space $\ell^{\infty}$ of bounded scalar sequences has SUB and SGHP. Observing that $\ell^{\infty}$ is the dual of $\left(\ell^{1}, \|\right.$. $\left.\|_{1}\right)$, we have the following.

Corollary 5. (Schur lemma [12], [1]) Every weakly convergent sequence in $\left(\ell^{1},\|\cdot\|_{1}\right)$ must be norm convergent.

Proof. Suppose $r^{n}=\left(r_{k}^{n}\right) \in \ell^{1}$ and $r^{n} \rightarrow 0$ weakly, i.e., for each $t=$ $\left(t_{k}\right) \in \ell^{\infty}, \lim _{n} r^{n} \cdot t=\lim _{n} \sum_{k=1}^{\infty} t_{k} r_{k}^{n}=0$. Let $\varepsilon>0$. By Theorem 1 , there is a $k_{0}$ such that $\left|\sum_{k=k_{0}+1}^{\infty} t_{k} r_{k}^{n}\right|<\varepsilon / 2$ for all $n$ and $\left(t_{k}\right)$ with $\sup _{k}\left|t_{k}\right| \leq 1$. On the other hand, for each $k, \lim _{n} r_{k}^{n}=0$ holds obviously and, hence, there is an $n_{0}$ 
such that $\sum_{k=1}^{k_{0}}\left|t_{k} r_{k}^{n}\right|<\varepsilon / 2$ for all $n>n_{0}$ and $\left(t_{k}\right)$ with $\sup _{k}\left|t_{k}\right| \leq 1$. Thus, $\left|\sum_{k=1}^{\infty} t_{k} r_{k}^{n}\right|<\varepsilon$ if $n>n_{0}$ and $\sup _{k}\left|t_{k}\right| \leq 1$, i.e., $\sum_{k=1}^{\infty}\left|r_{k}^{n}\right| \leq \varepsilon$ if $n>n_{0}$.

Let $\lambda$ be a family of scalar sequences. A sequence $\left\{x_{k}\right\}$ in a topological vector space $X$ is said to be $\lambda$-multiplier convergent $\left(\lambda\right.$-mc) if the series $\sum_{k=1}^{\infty} t_{k} x_{k}$ converges for each $\left(t_{k}\right) \in \lambda$. Recently, the first author has given the following nice result [7] :

Let $X$ be a Hausdorff locally convex space with the dual $X^{\prime}$ and $\lambda=c_{0}$ or $\ell^{p}(p \geq 1)$. Then $\lambda$-mc is an invariant for all admissible polar topologies, i.e., if $\left\{x_{k}\right\} \subseteq X$, then the series $\sum_{k=1}^{\infty} t_{k} x_{k}$ converges for each $\left(t_{k}\right) \in \lambda$ under the strongest admissible topology $\beta\left(X, X^{\prime}\right)$ if and only if $\sum_{k=1}^{\infty} t_{k} x_{k}$ converges for each $\left(t_{k}\right) \in \lambda$ under the weakest admissible topology $\sigma\left(X, X^{\prime}\right)$.

By Theorem 4 we can generalize this result. Recall that if $X$ is a barrelled space with the dual $X^{\prime}$, then the topology on $X$ is just $\beta\left(X, X^{\prime}\right)$; if $X$ is a bornological space and $Y$ is an arbitrary locally convex space, then every bounded linear operator $T: X \rightarrow Y$ is continuous and, hence, every sequentially continuous linear operator $T: X \rightarrow Y$ must be continuous. Note that a locally convex metric space is bornological and a barrelled bornological space need not be an inductive limit of Banach spaces ([4], p, 39).

Theorem 6. Let $(\lambda, \tau)$ be a barrelled bornological AK-space of scalar sequences such that $\tau$ is stronger than the topology of coordinatewise convergence and $(\lambda, \tau)$ has 0-GHP, e.g., $(\lambda, \tau)=\left(c_{0},\|\cdot\|_{\infty}\right)$ or $\left(\ell^{p},\|\cdot\|_{p}\right), p \geq 1$. If $\left\{x_{k}\right\}$ is a sequence in a Hausdorff locally convex space $X$ with the dual $X^{\prime}$ such that for each $\left(t_{k}\right) \in \lambda$ the series $\sum_{k=1}^{\infty} t_{k} x_{k}$ converges weakly, then for each $\left(t_{k}\right) \in \lambda$ the series $\sum_{k=1}^{\infty} t_{k} x_{k}$ converges under the strongest admissible topology $\beta\left(X, X^{\prime}\right)$.

Proof. Suppose that $t^{n}=\left(t_{k}^{n}\right) \rightarrow 0$ as $n \rightarrow+\infty$ in $(\lambda, \tau)$. Then for each $k, \lim _{n} t_{k}^{n}=0$ and, hence, $\lim _{n} t_{k}^{n} x_{k}=0$. Since $x=\left(x_{k}\right) \in \lambda^{\beta(X, \text { weak })}$ and the singleton $\{x\}=\left\{\left(x_{k}\right)\right\}$ is $w \lambda$-sequentially compact, by Theorem 4 the series $\sum_{k=1}^{\infty} t_{k}^{n} x_{k}$ converges in $(X$, weak) uniformly for $n \in \mathbb{N}$. Therefore, in $\left(X\right.$, weak) we have that $\lim _{n} \sum_{k=1}^{\infty} t_{k}^{n} x_{k}=\sum_{k=1}^{\infty} \lim _{n} t_{k}^{n} x_{k}=0$. Observing that $(\lambda, \tau)$ is bornological and letting $T\left(\left(t_{k}\right)\right)=$ weak- $\sum_{k=1}^{\infty} t_{k} x_{k}$, we obtain that $T$ : $(\lambda, \tau) \rightarrow(X$, weak $)$ is continuous and linear. Now let $\lambda^{\prime}=(\lambda, \tau)^{\prime}$. By the 
Hellinger-Toeplitz theorem ([16] , p. 168, Th. 2), T must be $\beta\left(\lambda, \lambda^{\prime}\right)-\beta\left(X, X^{\prime}\right)$ continuous. But $\beta\left(\lambda, \lambda^{\prime}\right)=\tau$ because $(\lambda, \tau)$ is barrelled. Thus $T$ is $\tau-\beta\left(X, X^{\prime}\right)$ continuous.

Now let $\left(t_{k}\right) \in \lambda$ be arbitrary. Since $(\lambda, \tau)$ is an AK-space, $\left(t_{1}, t_{2}, \ldots, t_{n}, 0\right.$, $0, \ldots) \stackrel{\tau}{\rightarrow}\left(t_{k}\right)$. Therefore,

$$
\sum_{k=1}^{n} t_{k} x_{k}=T\left[\left(t_{1}, \ldots, t_{n}, 0,0, \ldots\right)\right] \stackrel{\beta\left(X, X^{\prime}\right)}{\longrightarrow} T\left[\left(t_{k}\right)\right]=\text { weak- } \sum_{k=1}^{\infty} t_{k} x_{k},
$$

i.e., the series $\sum_{k=1}^{\infty} t_{k} x_{k}$ converges in $\left(X, \beta\left(X, X^{\prime}\right)\right)$.

\section{REFERENCES}

1. P. Antosik and C. Swartz, Matrix Methods in Analysis, Lecture Notes in Math., 1113, Heidelberg, 1985.

2. J.Boos and D. Fleming, Gliding hump properties and some applications, Internat. J. Math. Math. Sci. 1 (1995), 121-132.

3. J.Boos and T. Leiger, Some distinguished subspaces of domains of operator valued matrices, Results Math. 16 (1989), 199-211.

4. S. M. Khaleelulla, Counter Examples in Topological Vector Spaces, Lecture Notes in Math. 936, Heidelberg, 1982.

5. Lee Peng Yee, Sequence spaces and the gliding hump property, Southeast Asia Bull. Math. Special Issue (1993), 65-72.

6. Lee Peng Yee and C. Swartz, Continuity of superposition operators on sequence spaces, New Zealand J. Math. 24 (1995), 41-52.

7. Li Ronglu, An invariant with respect to all admissible topologies, to appear.

8. Li Ronglu and C. Swartz, Spaces for which the uniform boundedness principle holds, Studia Sci. Math. Hungar. 27 (1992), 379-384.

9. Li Ronglu and C. Swartz, A nonlinear Schur theorem, Acta Sci. Math. (Szeged) 58 (1993), 497-508.

10. D. Noll, Sequential completeness and spaces with the gliding humps property, Manuscripta Math. 66 (1990), 237-252.

11. D. Noll and W. Stadler, Abstract sliding hump technique and characterization of barrelled spaces, Studia Math. 94 (1989), 103-120.

12. J. Schur, Uber lineare Transformation in der Theorie die unendlichen Reihen, J. Reine Angew. Math. 151 (1920), 79-111.

13. C. Swartz, The gliding hump property in vector sequence spaces, Mh. Math. 116 (1993), 147-158. 
14. C. Swartz, Automatic continuity and boundedness of matrix mappings, Bull. Polish Acad. Sci. Math. 43 (1995), 19-28.

15. C. Swartz, Infinite Matrices and the Gliding Hump, World Scientific, Singapore, 1996.

16. A. Wilanski, Modern Methods in Topological Vector Spaces, McGraw-Hill, New York, 1978.

17. Wu Junde, Li Ronglu and C. Swartz, Continuity and boundedness for operatorvalued matrix mappings, to appear.

Li Ronglu

Department of Mathematics, Harbin Institute of Technology

Harbin, China

Min-Hyung Cho

Departmeht of Applied Mathematics, Kum-Oh University of Technology

Kumi, Korea 